\title{
Perspectivas da participação do fisioterapeuta no Programa Saúde da Família na atenção à saúde do idoso
}

\author{
Perspectives of physical therapy participation \\ in the Family Health Program for elderly care
}

M ariana Chaves Aveiro ${ }^{1}$ Giovanni Gurgel Aciole ${ }^{2}$ Patricia Driusso ${ }^{3}$ JorgeO ishi ${ }^{4}$
${ }^{1}$ Departamento deCiências daSaúde, Curso de

Fisioterapia, Universidade

Federal deSão Paulo,

Campus Baixada Santista.

Av. Alm. Saldanha da Gama

89, Ponta da Praia.

11030-400 Santos SP.

mariaveiro@yahoo.com

${ }^{2}$ Departamento de

M edicina, Universidade

Federal deSão Carlos.

${ }^{3}$ Departamento de

Fisioterapia, Universidade

Federal deSão Carlos.

${ }^{4}$ Departamento de

Estatística, Universidade

Federal de São Carlos.
Abstract The premise of this paper was to introduce the concept of elderly physical therapy care in the Family Health Program. Inspired by the impact attributable to the socio-demographic changes, theincrease of an elderly population and for the consolidation of a national health policy inspired by civil rights principles, the authors present an initiative for physical therapy practice aimed at the elderly. The succesfful integration of physical therapy in the Family $\mathrm{H}$ ealth Program conducted in the city of São Carlos, São Paulo State, has shown that the idea is likely to achieve great results and qualified participation. Key words Physical therapy (specialty), Elderly health care, Family health program
Resumo M obilizados pela problemática em torno das mudanças sociodemográficas, particularmente com o aumento da população de idosos, pela consolidação deuma política nacional desaúde calcada na noção de di reitos de cidadania ea partir das reflexões suscitadas pela experiência deSão Carlos (SP), na qual há a inserção do fisioterapeuta no PSF sob a forma de apoio matricial, os autores propõem, neste artigo, uma configuração do campo enúcleo das práticas de fisioterapia, na atenção à saúde do idoso, sob a perspectiva de sua inserção qualificada no Programa de Saúde da Família.

Palavras-chave Fisioterapia (especialidade), Saúde do idoso, Programa Saúde da Família 


\section{Introdução}

Vivemos o que vem sendo denominado de transição epidemiológica', na qual vem se observando o crescimento da população deidosos, deforma mais acentuada nos países em desenvolvimento. Segundo o Instituto Brasileiro de $\mathrm{Geo}$ grafia e Estatística (IBGE)², em relação aos países da América Latina, o Brasil assume uma posição intermediária, com uma população de idosos correspondendo a 8,6\% da população total. No período compreendido entre os censos de 1991 e 2000, por exemplo, o número de idosos aumentou em quase quatro milhões de pessoas.

Considerando a continuidade das tendências verificadas para as taxas de fecundidade e longevidade da população brasileira, as estimativas para os próximos vinte anos indicam que a população idosa poderá exceder trinta milhões de pessoas, chegando a representar quase $13 \%$ da população. Assim, embora a fecundidade ainda seja o principal componente da dinâmica demográfica brasileira, em relação à população idosa, é a longevidade que vem progressivamente definindo seus traços de evolução?

Decorrente desse processo, o país tem se mobilizado na definição de políticas públicas para este segmento, tendo elaborado a Política N acional de Saúde do Idoso, que assumiu como propósito a promoção do envelhecimento saudável, a manutenção ea melhoria, ao máximo, da capacidade funcional, a prevenção de doenças, a recuperação da saúde dos que adoecem e a reabilitação daqueles que venham a ter sua capacidade funcional restringida, de modo a garantir-Ihes a permanência no meio em quevivem, exercendo de forma independentesuas funçõesna sociedade ${ }^{3}$. Do mesmo modo, o Estatuto do Idoso, Lei no 10.741 de 01/10/2003, no seu Capítulo IV, artigo 15, assegura a atenção integral à saúde do idoso, por intermédio do Sistema Ú nico de Saúde (SUS), garantindo-Iheo acesso universal igualitário, em conjunto articulado e contínuo das ações e serviços, para a prevenção, promoção, proteção e recuperação da saúde, incluindo a atenção especial às doenças que afetam preferencialmente os idosos ${ }^{4}$.

No contexto da saúde mais geral, o Brasil, nas últimas décadas, vem conquistando importantes avanços. Regulamentado pela Constituição Federal de 1988 epelas Leis Complementares (Leis no 8.080/90 e n 8.142/90), o SU S ancora-se na definição de que "a saúdeé um direito fundamental do ser humano, devendo o Estado prover as condições indispensáveis ao seu pleno exercício". O processo de construção desta política de saúde vem gradativamente ocorrendo sobre os pilares da universalização, da integralidade, da descentralização e da partici pação popular 5 .

Como estratégia de consolidação destas políticas, o M inistério da Saúde assumiu, a partir de 1994, o Programa de Saúde da Família (PSF), visando à reorganização do modelo tradicional por intermédio da reorientação da atenção básica à saúde. A base da estratégia da saúde da família (Programa de Saúde da Família - PSF) é um modelo de atenção voltado à proteção e à promoção da saúde, além de atendimento domiciliar, com os seguintes aspectos organizacionais: delimitação de área de abrangência com adscrição da clientela (oitocentas a mil famílias por equipe); equipe multiprofissional (no mínimo médico, enfermeira, auxiliar de enfermagem e agentes comunitários); enfoque preventivo e de promoção à saúde, a partir de prioridades epidemiológicas da área adstrita; ênfase nas ações locais, visando reduzir a demanda sobre centros de saúde e hospitais; participação comunitária e controle social, centrados nos Consel hos M unicipais de Saúde 6 . A estratégia se integra numa rede deserviços, de forma quese garanta atenção integral aos indivíduos e famílias, assegurando-se a referência e contrarreferência para os diversos níveis do sistema, de problemas identificados na atenção básica. Entretanto, o modelo assistencial ainda predominanteno país é caracterizado pela prática médica voltada para uma abordagem biológica e intra-hospitalar, apresentando cobertura e resolubilidade baixase com elevado custo. 0 êxito da reforma proposta com o uso potencializado da atenção básica, complementada pela rede de serviços especializados e hospitalares, vem sendo a busca permanente dos gestores de saúde 5 .

N esse sentido, o PSF faria a promoção do acesso, prioritariamente, para os grupos sociais mais vulneráveis ${ }^{7}$, entre os quais estariam incluídos os idosos, os portadores de deficiência, as crianças e adolescentes e os adultos portadores de doenças crônico-degenerativas, por exemplo. Assim, de acordo com Silvestre e Costa N eto ${ }^{5}$, o PSF deve representar para o idoso, idealmente, 0 vínculo com o sistema de saúde por meio de uma equipe multiprofissional, que assume a responsabilidade por uma determinada população, em território definido, onde desenvolve suas ações, assumindo os compromissos de reconhecer a saúde como um direito de cidadania, humanizando as práticas de saúde e buscando a satisfação do usuário pelo seu estreito relacionamento com os profissionais de saúde; prestar assistência universal, integral, equânime, contínua e, aci- 
ma de tudo, resolutiva e de boa qualidade à população, na unidade de saúdeeno domicílio, elegendo a família, em seu contexto social, como núcleo básico de abordagem no atendimento à saúde; identificar os fatores de risco aos quais a população está exposta e neles intervir de forma apropriada; proporcionar o estabelecimento de parcerias pelo desenvolvimento de ações intersetoriais que visem à manutenção e à recuperação da saúde da população; estimular a organização da comunidade para o efetivo exercício do controlesocial.

A partir de 2005, o M inistério da Saúde favoreceu a ampliação da Atenção à Saúde da Família por meio da Portaria no 1065/GM , criando os Núcleos de Atenção I ntegral na Saúde da Família com a finalidade de ampliar a integralidade e a resolubilidade da atenção à saúde. A referida portaria, em seu artigo 70, definiu as modalidades e os profissionais responsáveis: alimentação/ nutrição e atividade física (nutricionista, educador físico e instrutor de práticas corporais); atividade física (educador físico e instrutor de práticas corporais); saúde mental (psicólogo, psiquiatra, terapeuta ocupacional e assistente social, sendo obrigatória a presença do psicólogo ou de psiquiatra e de pelo menos mais um profissional entre os mencionados); reabilitação (fisioterapeuta, fonoaudiólogo, terapeuta ocupacional e assistente social, sendo obrigatória a presença do fisioterapeuta e de pelo menos maisum profissional entre os mencionados) ${ }^{8}$.

Em 2006, por meio da Portaria no 687, o Ministério da Saúde aprovou a Política Nacional de Promoção da Saúde, cujo objetivo geral é "promover a qualidadedevida ereduzir vulnerabilidade e riscos à saúde relacionados aos seus determinantes e condicionantes: modos de viver, condições de trabalho, habitação, ambiente, educação, lazer, cultura, acesso a bens e serviços essenciais" ${ }^{\prime \prime}$.

Entretanto, a di visão dos N úcleos de Atenção Integral em categorias, que abrangeriam as áreas imprescindíveis, restringiu a atuação dos profissionais a uma ou duas categorias, desconsiderando parte da competência e habilidades dos mesmos para atuarem na atenção básica. Assim, possibilitou surgir uma nova proposta, os Núcleos de A poio à Saúde da Família (NASF).

Os NASF consistem em equipes compostas por profissionais de diferentes áreas de conhecimento que trabalharão em parceria com os profissionais das Equipes deSaúde da Família, compartilhando as práticas em saúde nos territórios sob responsabilidade das equipes, atuando diretamente no apoio às equipes e na unidade na qual o NASF será cadastrado. Os NASF serão compostos por no mínimo cinco profissionais de nível superior de diferentes ocupações, como médico acupunturista, assistente social, profissional da educação física, farmacêutico, fisioterapeuta, fonoaudiólogo, médico ginecologista, médico homeopata, nutricionista, médico pediatra, psicólogo, médico psiquiatra e terapeuta ocupacional (NASF 1) ou três profissionais de nível superior, entre assistente social, profissional de educação física, farmacêutico, fisioterapeuta, fonoaudiólogo, nutricionista, psicólogo e terapeuta ocupacional (NASF 2) ${ }^{10}$.

Assim, o fisioterapeuta insere-se no sistema de atenção básica numa nova perspectiva de atuação, focada nas práticas de prevenção e promoção e não restrita aos procedimentos de reabilitação, ao contrário do quetem predominado nas ações deste segmento profissional. Assim, este artigo tem como objetivo contribuir para a discussão da participação do fisioterapeuta na prevenção de agravos, promoção da saúde e recuperação da saúde dos principais problemas de saúde dos idosos.

\section{Inserção do fisioterapeuta no PSF}

A formação do fisioterapeuta tem sido voltada para a prática médica com uma abordagem biológica eintra-hospitalar. A ausência de definições do objeto de estudo da área de conhecimento e do objeto detrabal ho da profissão, aliados à atenção quase que exclusiva à doença, são potencializados pela sua própria gênese, evolução histórica, legislação e currículo de formação nos cursos de graduação $0^{11}$.

Entretanto, recentemente, a conjuntura de mudanças e transformações por que passa 0 campo das políticas e práticas de saúde, com o paulatino avanço de organização e efetivação do SUS, tem levado a fisioterapia a se inserir gradativamentena atenção básica, ampliando seu campo de atuação para além da reabilitação, com enfoque também para a prevenção de doenças e promoção de saúde. Embora a inserção na assistência básica não se apresenteainda como uma realidadenacional, o incremento de experiências municipais revelam um crescimento da atuação do fisioterapeuta no SU S com o apoio dos gestores locais.

Autores como Pereira et al. ${ }^{12}$ descrevem o objeto de ação do fisioterapeuta no PSF como "movimento humano visando à saúde funcional do indivíduo na promoção erecuperação da saú- 
de, prevenção de doenças e agravos e reabilitação" e o objetivo geral da sua atuação como o de "promover a qualidade de vida do indivíduo, em todos os ciclos da vida tendo a integridade do movimento como essência eexpressão desta, por meio de cinesioterapia, recursos físicos e naturais no PSF".

0 fisioterapeuta deveria, portanto, articular suas ações integrando a recuperação, a prevenção de incapacidades e/ ou doenças ea promoção da saúde, intervindo não só no indivíduo, mas também no coletivo. Programar suas ações levando em consideração os aspectos sociais, econômicos, culturais e ambientais que podem intervir no processo saúde-doença. As visitas domiciliares que realiza devem ter uma abordagem familiar, não centrada no indivíduo acometido por alguma doença, mas compartilhar a responsabilidade da intervenção com todos os membros, buscando soluções mais eficientes e próximas da realidade da família ${ }^{13}$.

As atividades que os fisioterapeutas vêm realizando no PSF, desenvolvidas nas Unidades de Saúde da Família (USF) e em domicílio,o são, dentre outras, reconhecimento da área descentralizada, potencialidades da comunidade, grupos de gestantes, grupos de postura, grupos de mães de crianças com infecção respiratória aguda, grupo deprevenção deincapacidades em hanseníase, grupo de mães de crianças com problemas neurológicos, grupo de idosos, atuação no climatério, atuação na saúde da criança, atendimento individual, estimulação essencial em crianças com atraso no desenvolvimento neuropsicomotor, atuação nas creches, reeducação postural, acompanhamento de pacientes acometidos pela hanseníase, resgate dos cuidadores dentro do ambiente familiar e orientações de saúde em geral ${ }^{14}$.

Em Londrina (PR), por exemplo, a fisioterapia ainda não está, oficialmente, incluída no PSF e enfrenta alguns entraves. 0 desenvolvimento de ações preventivas e educativas não constitui prioridade devido à grande demanda por atendimento de reabilitação, há um reduzido número de profissionais atuando no PSF e a infraestrutura (espaço físico erecursos terapêuticos) das UBS não é adequada para a assistência fisioterapêutica ${ }^{15}$.

Em São Carlos (SP), a inserção está ocorrendo por meio da Residência Multiprofissional em Saúde da Família e Comunidade da Universidade Federal de São Carlos, desde 2007, na forma de apoio matricial. 0 contato do profissional com o usuário tem ocorrido pelo encaminha- mento da demanda espontânea e/ou a corresponsabilização na produção de cuidado via discussão de situações de indivíduos e/ou famílias. Há grande demanda de usuários que necessitam de cuidado relacionado à dor eà limitação física, remetendo, o fisioterapeuta, ao estigma de reabilitação e ao olhar reducionista do indivíduo. A pesar deste cenário, algumas ações desenvolvidas propõem a reorganização do serviço, dentre elas a participação em atividades já existentes ( grupo de usuários com al gia de coluna, gestante, atividade física, acolhimento, visita domiciliar, puericultura, reuniões de conselho gestor) ea construção de novos projetos (grupo de usuários de psicotrópicos, idosos, sala de espera, periódicos da unidade, parcerias/integração com outros equipamentos sociais, elaboração de eventos, proposição de práticas corporais não convencionais, diagnóstico do território).

\section{Atenção à saúde do idoso no contexto do Programa de Saúde da Família}

De acordo com a Política Nacional de Saúde do Idoso, o principal problema que pode afetar 0 idoso, como consequência da evolução de suas enfermidades e de seu estilo de vida, éa perda de sua capacidade funcional, isto é, a perda das habilidades físicas e mentais necessárias para a realização de suas atividades básicas e instrumentais da vida diária3.

A capacidade funcional tem sido considerada um novo paradigma de saúde, particularmente relevante para o idoso. Envelhecimento saudável, dentro dessa nova ótica, tem sido resultante da interação multidimensional entre saúde física, saúdemental, independência na vida diária, integração social, suporte familiar e independência econômica. 0 bem-estar na vel hiceou saúdenum estado mais amplo seria o resultado do equilíbrio entre as várias dimensões da capacidade funcional do idoso, sem necessariamente significar ausência de problemas em todas as dimensões ${ }^{16}$.

Um idoso com uma ou mais doenças crônicas pode ser considerado um idoso saudável, se comparado a idosos com as mesmas doenças, porém sem controle destas, levando a sequelas e incapacidades associadas ${ }^{16}$. Utilizando dados da Pesquisa Nacional por Amostra de Domicílios (PNAD), Lima-Costa et al. ${ }^{17}$ investigaram indicadores gerais de saúde da população idosa brasilei ra everificaram que cerca de $70 \%$ dos idosos relataram ter pelo menos uma condição crônica, sendo esta proporção maior entre mulheres 
$(74,5 \%)$ do que entre os homens $(62,2 \%)$ e aumentando com a idade em ambos os sexos.

A adoção do modelo de cuidados domiciliares pode favorecer a manutenção da capacidade funcional, já que possibilita ao idoso o convívio social, interação com meio ambiente e estímulos físicos ementais. Asinstituições delonga permanência constituem-se, na mai oria das vezes, opção ímpar para esses indivíduos; no entanto, favorecem o isolamento, inatividade física e mental, tendo, dessa forma, consequências negativas à qualidade devida ${ }^{18}$. A assistência domiciliar aos idosos com comprometimento funcional demanda programas de orientação, informação e apoio de profissionais capacitados em saúde do idoso edepende, essencialmente, do suporteinformal e familiar, constituindo-se num dos aspectos fundamentais na atenção à saúde desse grupo populacional. Isso não significa, no entanto, que o Estado deva deixar de ter um papel preponderante na promoção, proteção e recuperação da saúde do idoso 5 .

Para o al cancedo propósito da Política Nacional do Idoso ${ }^{3}$, foram definidas como diretrizes essenciais à promoção do envelhecimento saudável a manutenção da capacidade funcional, a assistência às necessidades de saúde do idoso, a reabilitação da capacidadefuncional comprometida, a capacitação de recursos humanos especializados, o apoio ao desenvolvimento de cuidados informais e 0 apoio a estudos e pesquisas.

A manutenção da capacidade funcional pode ter importantes implicações para a qualidade de vida dos idosos, por estar relacionada com a capacidade de ocupar-se com o trabal ho atéidades mais avançadas e/ou com atividades agradáveis. Parece, portanto, bastanterelevante planejar programas específicos de intervenção para eliminação de certos fatores de risco relacionados com a incapacidade funcional ${ }^{19} \mathrm{e}$, consequentemente, melhora da qualidade da vida adicional adquirida ao longo das décadas, com o aumento da longevidade ${ }^{20}$.

O Programa de Saúde da Família (PSF) tem revelado uma dupla funcionalidade: é tanto um mecanismo indutor da equidade no cuidado em saúde para os idosos, por alcançar mais aquelas populações que socialmente mais necessitam, quanto tem sido uma forma mais efetiva de promover o cuidado ${ }^{21}$, abordando mudanças físicas consideradas fisiológicas e identificação precoce de suas alterações patológicas. 0 estudo de Piccini et al. ${ }^{21}$ ressalta, além disso, a importância de se alertar a comunidade sobre os fatores de risco a que as pessoas idosas estão expostas, no domicí- lio e fora dele, bem como de serem identificadas formas de intervenção para sua eliminação ou minimização, sempre em parceria com o próprio grupo de idosos e os membros de sua família. Os profissionais que atuam na aten ção básica devem ter de modo claro a importância da manutenção do idoso na rotina familiar ena vida em comunidade como fatores fundamentais para a manutenção de seu equilíbrio físico emental ${ }^{5}$. A equipe de saúde precisa estar sempreatenta à pessoa idosa, na constante atenção ao seu bem-estar, à sua rotina funcional eà sua inserção familiar e social, jamais a deixando à margem de seu contexto, mantendo-a o mais independentepossível no desempenho de suas atividades rotineiras ${ }^{22}$.

São competências, habilidades e atribuições comuns da equipe da atenção básica sob o PSF voltadas à pessoa idosa, segundo Silvestre e Costa $\mathrm{N}$ et $0^{5}$, o conhecimento da realidade das famílias pelas quais são responsáveis, com ênfase nas suas características sociais, econômicas, culturais, demográficas e epidemiológicas; a identificação dos problemas de saúde e situações de risco mais comuns aos quais o idoso está exposto e a elaboração de um plano local para o enfrentamento dos mesmos; a execução, de acordo com a formação e qualificação de cada profissional, dos procedimentos de vigilância à saúde da pessoa idosa; a valorização das relações com a pessoa idosa e sua família, para a criação de vínculo de confiança, de afeto e de respeito; a realização de visitas domiciliares de acordo com o planejado; a prestação de assistência integral à população idosa, respondendo às suas reais necessidades deforma contínua eracionalizada; a garantia de acesso ao tratamento dentro de um sistema de referência e contrarreferência para aqueles com problemas mais complexos ou que necessitem de internação hospitalar; a coordenação e participação e/ ou organização degrupos de educação para a saúde; a promoção de ações intersetoriais e de parcerias com organizações formais e informais existentes na comunidade para o enfrentamento conjunto dos problemas identificados na população idosa, além da fomentação da participação popular, discutindo com a comunidade conceitos de cidadania, de direitos à saúde e suas bases legais.

SilvestreeCosta eto $^{5}$ ressaltam quegeralmente o impacto individual dos problemas de saúde éa piora da qualidade de vida, com maior risco de dependênciaeaumento demorbimortalidadeeque os resultados esperados com a atuação da Equipe deSaúde da Família são a melhora da qualidadede vida, prevenção de complicações e dependência e menor número de hospitalizações. 
Diversos são os problemas de saúde de idososquepodem ser abordados pela equipede saúde da família, No Q uadro 1, foram incluídos, em grandes áreas de atuação, os problemas de saúde de idosos, que tem possibilidades de serem abordados no PSF pelo fisioterapeuta.

\section{Partici pação dafisioterapia para prevenção de agravos, promoção da saúde ereabilitação}

Como tem sido proposto nas atuais políticas de saúde no Brasil, não só o fisioterapeuta, mas também todos os profissionais de saúde que se propõem a atuar no PSF, devem valer-se de uma prática voltada para a prevenção de agravos, promoção da saúde e recuperação da saúde (tratamento ou reabilitação). Considerando os problemas de saúde dos idosos apresentados no Quadro 1, pode-se abrir amplas possibilidades de atuação para o fisioterapeuta no PSF.

No quese refereà prevenção de agravose promoção da saúde, de um modo geral, o fisioterapeuta podeestar contribuindo na identificação de grupos vulneráveis da área de atuação e de fatores de risco para doenças crônicas; na investigação de evidências da efetividade de ações de práticas de cinesioterapia/atividade física e recursos analgésicos no controle e prevenção de doenças crônicas; em campanhas de estímulo a modos de viver saudáveiscom objetivo de reduzir fatores de risco; na oferta de suporte e orientações a familiares e cuidadores na prevenção de quedas, incapacidades edeformidades; na capacitação da Equipe de Saúde da Família, no que concerne às suas habilidades ecompetên cias profissionais; na articulação com gestores para mobilização de recursos e fortalecimento de ações para um estilo de vida saudável, construção de espaços para práticas de atividade física/cinesioterapia e educação em saúde e na mobilização da comunidade para transformação do ambiente para condições favoráveis à saúde e acessibilidade a edificações, mobiliários e espaços urbanos, etc.

No que concerne às grandes áreas de atuação, formadas a partir dos principais problemas de saúde dos idosos, podem surgir novas possibilidades de contribuições, específicas do fisioterapeuta, tanto para prevenção de agravos e promoção da saúde, quanto para reabilitação.

Pensando em prevenção de agravos e promoção da saúde para problemas relacionados ao sistema musculoesquelético, por exemplo, o fisioterapeuta poderia contribuir na estimulação e fa- vorecimento de práticas constantes, em grupos, decinesioterapia/atividadefísica, tais como alongamento, fortalecimento muscular, treino deequilíbrio e caminhadas; na promoção de ações de reeducação postural, como as escolas de postura, ena assistência a alterações de ambientes e mobiliários para favorecer a acessibilidade, evitar acidentes como quedas ediminuir prevalência delesões crônicas laborais. $\mathrm{N}$ a reabilitação, entretanto, pode formar grupos para práticas de cinesioterapia, que incluam treinamento de resistência, equilíbrio e flexibilidade. Poderia, ainda, realizar atendimento fisioterapêutico individual na unidade, cinesioterapia e recursos eletrotermoterápicos, ou domiciliar, especialmente a idosos acamados; encaminhar para clínica de referência, quando houver necessidade de atendimento especializado, e favorecer próteses e órteses.

Em relação às doenças que compõem o grupo neurologia, no que concerne à prevenção de agravos e promoção da saúde, o fisioterapeuta poderia contribuir para um estilo de vida saudável, favorecendo práticas constantes de cinesioterapia/atividade física, ou seja, práticas dealongamento, fortalecimento muscular, treino de equilíbrio e caminhadas. Poderia, ainda, estimular a al imentação saudável, para controledo peso, do colesterol e da glicemia, e o controle do tabagismo e alcoolismo. Já na reabilitação, teria possibilidades de realizar atendimento fisioterapêutico individual na unidade ou domiciliar, especialmente a idosos acamados, por meio de cinesioterapia e estimulação cognitiva, podendo utilizar-se de técnicas específicas como Kabat, Bobath, etc.; de orientar cuidadores quanto à prevenção dedeformidades, feridas eúlceras; deformar grupos para práticas de cinesioterapia; de encaminhar para clínica de referência, quando necessário atendimento especializado, e defavorecer o acesso a órteses. Quando pressentir alterações sensoriais, apesar das limitações inerentes à sua formação profissional, pode contribuir no acoIhimento e no acesso a profissional especializado para exames e tratamento, além de possível participação em campanhas para identificação de deficiências visuais e auditivas, com instrumentos específicos, e promoção de acesso a tratamento cirúrgico ou órteses corretivas.

No quese refereà saúdemental, para prevenção de agravos e promoção da saúde, o fisioterapeuta poderia participar da promoção de práticas de cinesioterapia/atividade física, de atividades lúdicas, de memória e de concentração, em grupo, quefavoreçam a social ização. Poderia, ainda, estimular a higiene do sono e articular com 
Quadro 1. Problemas de saúde apresentados por idosos passíveis de intervenção pelo fisioterapeuta no PSF.

\begin{tabular}{|c|c|}
\hline Área de atuação & Problemas de saúde \\
\hline Musculoesquelético & $\begin{array}{l}\text { Artrite reumatóide } \\
\text { Dorsalgia } \\
\text { Diminuição da capacidade funcional } \\
\text { Fraturas (colo de fêmur, rádio, vértebras) } \\
\text { Inatividade crônica } \\
\text { Osteoartrite } \\
\text { Osteopenia/osteoporose } \\
\text { Síndrome do imobilismo } \\
\text { Quedas }\end{array}$ \\
\hline N eurologia e alterações sensoriais & $\begin{array}{l}\text { AVE } \\
\text { Disfunção vestibular } \\
\text { Déficit auditivo } \\
\text { Déficit visual } \\
\text { Pakinsonismo } \\
\text { Tumores cerebrais }\end{array}$ \\
\hline Saúde mental & $\begin{array}{l}\text { Alucinação } \\
\text { Demência } \\
\text { Depressão maior } \\
\text { Distúrbios do sono } \\
\text { Isolamento }\end{array}$ \\
\hline Cardiovascular & $\begin{array}{l}\text { Cardiopatia isquêmica } \\
\text { Hipertensão arterial sistêmica } \\
\text { Hipertensão sistólica isolada } \\
\text { Insuficiência cardíaca congestiva } \\
\text { Insuficiência venosa crônica }\end{array}$ \\
\hline Respiratória & $\begin{array}{l}\text { Doença pulmonar obstrutiva crônica } \\
\text { Gripe } \\
\text { Pneumonia } \\
\text { Resfriado }\end{array}$ \\
\hline Disfunções endócrinas e nutricionais & $\begin{array}{l}\text { Desnutrição } \\
\text { Diabetes mellitus } \\
\text { Dislipidemia } \\
\text { Hipotiroidismo } \\
\text { Obesidade }\end{array}$ \\
\hline Ginecologia, Urologia e Coloproctologia & $\begin{array}{l}\text { Alterações do climatério } \\
\text { Constipação } \\
\text { Disfunção erétil e ejaculatória } \\
\text { Dispareunia, vaginismo } \\
\text { Distopia genital } \\
\text { Infecções sexualmente transmissíveis (IST) } \\
\text { Incontinência fecal } \\
\text { Incontinência urinária }\end{array}$ \\
\hline Oncologia & $\begin{array}{l}\text { Colo de útero } \\
\text { Colorretal } \\
\text { Mama } \\
\text { Próstata } \\
\text { Traquéia, brônquios, pulmões }\end{array}$ \\
\hline Cuidados paliativos & $\begin{array}{l}\text { Dor } \\
\text { Fragilidade }\end{array}$ \\
\hline Saúde coletiva & $\begin{array}{l}\text { Abuso, maus tratos, negligência } \\
\text { Alcoolismo } \\
\text { latrogenia, multifármacos } \\
\text { Tabagismo }\end{array}$ \\
\hline
\end{tabular}

N ota: Elaboração dos autores, com baseem Silvestree Costa N eto ${ }^{5}$. 
gestores a construção de espaços urbanos de convivência. Durantea reabilitação, deveria favorecer a adesão a grupos de práticas de cinesioterapia, com objetivo não só de recuperação funcional, mas também de favorecer a socialização, além de atendimento domiciliar ou individual na unidade de pacientes com maiores limitações funcionais. Seria prudente, ainda, se fornecesse adequado suporte a familiares e cuidadores, com orientação para estimulação das atividades cognitivas remanescentes e fizesse o referenciamento, para acompanhamento especializado.

Em relação às doenças do sistema cardiovascular, para a prevenção de agravos e promoção da saúde, o fisioterapeuta pode contribuir para um estilo de vida saudável, promovendo práticas constantes e regulares de cinesioter apia/atividade física (principalmente treinamento aeróbio como caminhadas), além de estimular o controle do tabagismo e alcoolismo euma al imentação saudável para controle do peso, do colesterol e da glicemia. Durantea reabilitação, podeparticipar da supervisão de grupos para práticas de cinesioterapia, que incluam treinamento aeróbio, alongamentos e fortalecimento muscular, além de realizar atendimento fisioterapêutico individual na unidade, ou referenciar para atendimento especializado idosos de mai or risco (Fases II e III de reabilitação cardiovascular). D eve-se sempre cuidar para prevenção de edemas e/ou linfedemas em membros inferiores, seja realizando drenagem linfática e enfaixamentos compressivos ou orientando para o uso de meias elásticas compressivas, quando indicados. Pode-se, ainda, aplicar recursos eletrotermoterápicos que auxiliem a cicatrização deferidas, ou encaminhar para atendimento especializado.

A prevenção de doenças e promoção da saúde em doenças respiratórias deveriam constar de divulgação e estimulação à vacinação em campanhas (uma dose anual da vacina contra influenza no outono e ao menos uma dose de vacina antipneumocócica durante a vida); favorecimento de estilo de vida saudável como práticas constantes e regulares de cinesioterapia/atividadefísica, alimentação saudável, controle do tabagismo e alcoolismo; educação para higienebrônquica e da orofaringe adequada e uma busca contínua junto à comunidadee gestores de estratégias para ambientes com menores índices poluição. No que concerne à reabilitação, poderia ser interessante formar grupos para práticas de cinesioterapia, por meio detreinamento aeróbio, erealizar atendimento domiciliar enaunidade dehigienebrônquica e treinamento muscular respiratório, além de favorecer esupervisionar a suplementação de oxigênio domiciliar, quando necessário, e encaminhar para atendimento especializado em clínica de referência.

Refletindo sobre as disfunções endócrinas e nutricionais, nota-se a importância na participação durante o planejamento e promoção de ações para a segurança alimentar e nutricional. Pode-se, ainda, contribuir ofertando ações de práticas de cinesioterapia/atividadefísica; estimulando ações relativas à alimentação saudável em atendimentos individuais, atividades em grupo e visitas domiciliares; participando de ações de vigilância alimentar nutricional earticulando com a equipe e gestores sobre a qualidade nutricional para as refeições. Em relação à reabilitação, sugere-se que o fisioterapeuta poderia formar grupos de práticas de cinesioterapia, principalmente treinamento aeróbio; realizar atendimento fisioterapêutico individual na unidade ou referenciar para atendimento especializado idosos com presença de comorbidades; acompanhar idosos re ferenciados; intervir sobre doenças decorrentes; estimular ações rel ativas à alimentação sau dável; identificar sinais ou sintomas de alterações cognitivas, dermatológicas e/ ou funcionais e intervir sobre os mesmos por meio de cinesioterapia, outros recursos físicos e estimulação cognitiva.

Dentro das áreas deatuação ginecologia, urologia e coloproctologia, deve-se estimular um estilo de vida saudável com alimentação saudável, controle do tabagismo e alcoolismo e práticas constantes eregulares de cinesioterapia/atividade física, que poderiam incluir, além de fortalecimento muscular, atividades aeróbias e alongamentos e o treinamento dos músculos do assoalho pélvico. A participação em campanhas de divulgação objetivando transformar comportamentos individuais, por exemplo, micção programada; a abordagem na avaliação geriátrica da sexualidade; a identificação defatores derisco para disfunção sexual (fisiológicos e/ou psicológicos); 0 encaminhamento para avaliação médica e/ou psicológica para tratamento adequado de casos identificados; o favorecimento ao acesso e estímulo ao uso correto de preservativos e lubrificantes e 0 encaminhamento para o exame deinfecções sexualmentetransmissíveis(IST) são as demais ações em que o fisioterapeuta poderia, em muito, contribuir para a prevenção de agravos e promoção da saúde. Em relação à reabilitação, poder-se-iam formar grupos para práticas de cinesioterapia, especialmente de treinamento muscular do assoalho pélvico e dos abdominais, com uma abordagem comportamen- 
tal para micção programada e hábitos alimentares saudáveis, além de realizar atendimento individual na unidade; estimular a adesão a terapia antirretroviral; oferecer atendimento individual na unidade, para a doença associada, e encaminhar para clínica de referência para atendimento especializado quando necessário.

No que concerne à oncologia, dever-se-ia favorecer 0 acesso ao diagnóstico precoce, por meio do exame Papanicolau, autoexame da mama, mamografias, toque retal, etc.; estimular estilo de vida saudável com práticas constantes e regulares decinesioterapia/atividadefísica, alimentação saudável, controle do tabagismo e alcoolismo e participar de campanhas educativas. Durante a reabilitação, o fisioterapeuta poderia ser o responsável por formar grupos de práticas de cinesioterapia, para manutenção da capacidade funcional, além de oferecer atendimento domiciliar ou individual na unidade para incapacidades funcionais decorrentes do câncer e de seu tratamento; informar quanto à prevenção de complicações decorrentes de linfadenectomia; fazer orientações prée pós-operatórias; realizar atendimento pós-operatório ou encaminhar para clínica de referência para atendimento especializado; dar suportee/ou orientar familiares e cuidadores; e conscientizar sobre os direitos do paciente com câncer.

Em ocasiões de estados terminais, poderia também caber ao fisioterapeuta favorecer cuidados paliativos, como aliviar a dor e outros sintomas angustiantes, melhorando a qualidade de vida, além de contribuir para afirmar a vida e considerar o morrer um processo natural; favorecer ajuda para viver tão ativamentequanto possível atéa morte; efavorecer um sistema de suporte à família para lidar com a doença e com o luto.

Quando constatada uma demanda em alcoolismo, o fisioterapeuta, em parceria com a equipe, pode participar da promoção de ações educativas e sensibilizadoras quanto ao uso abusivo deálcool esuas consequências; do apoio a políticas de restrição de acesso a bebidas alcoólicas, protegendo segmentos vulneráveis e priorizando situações de violência e danos sociais; decampanhas locais, em interação com as agências de trânsito, no alerta quanto às consequências da "direção alcoolizada"; da identificação de sinais de alterações funcionais, cognitivas e de doença hepática, com posterior encaminhamento para avaliação médica; do desenvolvimento de ações voltadas para um estilo devida saudável; da oferta de ações de práticas de cinesioterapia/atividade física; e da articulação com gestores sobre a importância de desenvolver políticas relaciona- das a disponibilidade de álcool. No que tange à reabilitação, pode contribuir intervindo sobre doenças decorrentes, por meio de cinesi oterapia e outros recursos físicos, formando grupos de práticas de cinesioterapia e realizando atendimento fisioterapêutico individual na unidade, com objetivo de melhora da capacidade funcional.

Entretanto, se prevalente o tabagismo, o fisioterapeuta poderia contribuir no apoio aos fumantes para a cessação de fumar; na redução da aceitação social do tabagismo; na proteção da população aos riscos da exposição à poluição tabagística ambiental; na manutenção deum fluxo contínuo de informações sobre o tabagismo, seus riscos para quem fuma e os riscos da poluição tabagística ambiental para todos; e no de senvolvimento de ações voltadas para um estilo de vida saudável, principalmente favorecendo práticas decinesioterapia/atividadefísica. Em re lação à reabilitação, pode estar presente formando grupos para práticas de cinesioterapia e realizando atendimento individual defisioterapia respiratória na unidade, com objetivo de melhora da capacidade funcional.

O fisioterapeuta, se em contato diário com o idoso, deve estar sempre atento a possíveis maus tratos para identificação e encaminhamento adequado deviolência intrafamiliar, negligênciaeabuso. Deve-se, também, implementar a ficha denotificação de violência interpessoal; envolver a comunidade em ações de proteção ao idoso; articular com gestores para mobilizar recursos com objetivo de monitorar e controlar situações de abuso, maus tratos e negligência, além de manter a capacidadefuncional do idoso por meio deações de práticas de cinesioterapia/atividadefísica edar suporte a familiares e cuidadores. Durante a reabilitação, poderealizar atendimento individual na unidadeou domiciliar decinesioterapia e/ou analgesia para reabilitação de consequências funcionais edar suporte eorientar familiares e cuidadores e, sendo necessário, encaminhar para atendimento especial izado em clínica de referência.

Sendo a iatrogenia e multifármacos prevalentes em idosos, decorrentes da incidência de mais de uma doença crônica, identificar sinais de interação entre medicamentos e efeitos adversos; referenciar para o atendimento médico para estabelecimento de prioridades; promover esclarecimentos sobre os riscos da automedicação em atendimentosindividuais, atividades em grupo e visitas domiciliares; e ofertar ações de atividade física que possam substituir a necessidade de maior número de medicamentos são ações importantes que deveriam fazer parte do campo de 
atuação do fisioterapeuta para a prevenção de agravos e promoção da saúde. Enquanto, na reabilitação, propõe-se realizar atendimento individual na unidade ou domiciliar para reabilitação de consequências funcionais e encaminhar para clínica de referência, quando houver presença de comorbidades.

\section{Discussão}

Com a crescente demanda de fisioterapeutas na atenção básica, faz-se necessário definir a atuação desse profissional. 0 mesmo tem amplas possibilidades de atuação, que ainda não são completamente exploradas, quando atuando no PSF, por dificuldades de definição de seu campo de trabalho. As ações ainda estão restritas a algunsmunicípios, principalmenteno queconcerneà preven ção de agravos e promoção da saúde.

Silvestre e Costa $\mathrm{N} \mathrm{eto}^{5}$ levantaram uma série de problemas de saúde que necessitam de atenção para a saúde do idoso; assim, foi proposto refletir sobre as contribuições do fisioterapeuta nesses processos mais prevalentes entre os idosos sob a responsabilidade de cuidados da família e atenção pela equipe de saúde da família.

Para alcançar estepropósito, optou-se em classificar os problemas desaúde dosidosos, em grandes áreas de atuação, nas quais a ação do fisioterapeuta tanto para prevenção de agravos, quanto para promoção da saúde, assemelhavam-se. Algumas ações, entretanto, diferiram-se muito das demais, sendo agrupadas em um grande grupo denominado saúde coletiva, e foram discutidas separadamente. Foram os problemas de saúde alcoolismo, tabagismo, maus tratos/abuso/negligência eiatrogenia/multifármacos, em queas principais ações, relacionadas, principalmente, à prevenção de agravos e promoção da saúde, compreendiam aquelas referentes à formação comum de profissionais de saúde, incluídos neste grupo. Dificuldades também foram encontradas para classificar déficits auditivo evisual, queacabaram sendo incluídos na categoria neurologia e al terações sensoriais, devido a prováveis alterações neurológicas que contribuem para esses déficits.

Para determinar as ações, gerais e especificas, para a prevenção de agravos e promoção da saúde, considerou-se uma definição ampla de promoção de saúde. A promoção da saúde consiste nas atividades dirigidas à transformação dos comportamentos dos indivíduos, focando nos seus estilos de vida e localizando-os no seio das famílias e, no máximo, no ambiente das culturas da comunidadeem que se encontram. Nestecaso, os programas ou atividades de promoção da saúdetendem a concentrar-seem componentes educativos, primariamente relacionados com riscos comportamentais passíveis de mudanças, que estariam, pelo menos em parte, sob o controle dos próprios indivíduos. Por exemplo, o hábito de fumar, a dieta, as atividades físicas, a direção perigosa no trânsito. E o que vem caracterizar a promoção da saúde, modernamente, é a constatação do papel protagonista dos determinantes gerais sobre as condi ç̧ões de saúde. Suas atividades estariam, então, mais voltadas ao coletivo de indivíduos e ao ambiente, compreendido num sentido amplo, de ambiente físico, social, político, econômico e cultural, por meio de políticas públicas e de condições favoráveis ao desenvolvimento da saúde (as escolhas saudáveis serão as mais fáceis) e do reforço (empowerment) da capacidade dos indivíduos e das comunidades ${ }^{23}$.

Para promoção da saúde, também se considerou a Política de Promoção da Saúde e suas ações específicas para al imentação saudável, prática corporal/atividade física, prevenção e controle do tabagismo e redução da morbimortalidade em decorrência do uso abusivo de álcool e outras drogas. Entretanto, em sua grande maioria, as ações do fisioterapeuta, específicas, para prevenção de agravos e promoção da saúde, também foram determinadas a partir dos principais fatores de risco para as doenças da área discutida. Foram considerados fatores de risco para doenças respiratórias, por exemplo, os mesmos considerados por Gomes ${ }^{24}$ para pneumonias adquiridas na comunidade: envelhecimento, tabagismo/D POC, insuficiência cardíaca, colonização da orofaringe, micro e macroaspiração, alcoolismo/cirrosehepática, deficiência nutricional, imunossupressão e fatores ambientais.

Para determinar a reabilitação para as diversas áreas, fez-se necessário pesquisar, além dos fatores de risco, sinais e sintomas decorrentes dos problemas de saúde apresentados. França e Tavares ${ }^{25}$, por exemplo, relatam presença de edema, linfedema, alterações tróficas e úlceras em pacientes com diagnóstico de insuficiência venosa crônica, dependendo do estágio de evolução. Assim, foram propostas alternativas deintervenção do fisioterapeuta para essas condições. 


\section{Considerações finais}

Decorrente do exposto, nota-se amplas possibilidades de participação qualificada, para o fisioterapeuta, na atenção à saúde do idoso, seja na prevenção de doenças e na promoção da saúde, seja na clássica reabilitação, utilizando-se de ferramentas de atuação específicas de sua formação profissional, como cinesioterapia e recursos eletrotermoterápicos.

Como integrante do NASF, sua atuação resulta ampliada, tendo em vista que realize diagnóstico denecessidadeseorganizedemanda; promova a conscientização da população para mudança do enfoque do tratamento; demedicamentos para tratamento por meio de recursos físicos; busque parcerias para intervenção em problemas desaúdeeenfrentamento destes; encaminhepara as clínicas de referência para tratamento especializado, não disponível na unidade; realize atendi- mento individual, na unidade edomiciliar; organizegrupos deações de práticas de cinesioterapia/ atividade física e contribua para a elaboração e implantação de políticas públicas integradas que visem à melhoria da qualidade de vida no planejamento de espaços urbanos e rurais.

Este conjunto de ações a desempenhar coloca sob exame sua formação como profissional de saúde. Se o ensino da profissão ainda é predominantemente centrado no enfoque biológico e na prática intra-hospitalar, a continuada inserção e inclusão do fisioterapeuta nas práticas assistenciais do PSF permite-nos pensar a possibilidade de que se amplifique o debate em torno das mudanças na formação profissional. Pode melhorar sua qualificação para atuação na atenção básica para que tenhamos fisioterapeutas que aliem boa qualidade técnica à adequação à realidade social do país e ao compromisso social com a saúde como direito de cidadania.

\section{Colaboradores}

MC Aveiro, GG Aciole eP Driusso participaram do levantamento bibliográfico, concepção, redação e revisão do texto. J Oishi participou da concepção e revisão do texto. 


\section{Referências}

1. Monteiro CA, organizador. Velhos e novos males de saúde no Brasil. São Paulo: Hucitec; 2000.

2. Instituto Brasileiro de Geografia e Estatística. Estudos e pesquisas de informação demográfica e econômica. Perfil dos idosos responsáveis pelos domicílios no Brasil. Rio de Janeiro: IBGE; 2002.

3. Brasil. Ministério da Saúde. Portaria n 1.395 de 9 de dezembro de 1999. Aprova a Política Nacional de Saúde do Idoso e dá outras providências. Diário Oficial da União 1999; 13 dez.

4. Brasil. M inistério da Saúde. Estatuto do Idoso. 1a ed. Brasília: M inistério da Saúde; 2003.

5. Silvestre JA, Costa Neto MM. Abordagem do idoso em programas de saúde da família. Cad Saude Publica 2003; 19(3):839-847.

6. Trad LAB, Bastos ACS O impacto sócio-cultural do Programa de Saúde da Família (PSF): uma proposta de avaliação. Cad Saude Publica 1998; 14(2):429-435.

7. Senna MCM. Equidade e política de saúde: algumas reflexões sobre o Programa Saúde da Família. Cad Saude Publica 2002; 18(Suppl.):203-211.

8. Brasil. Ministério da Saúde. Portaria n 1.065 de 4 de julho de 2005. Cria os Núcleos de Atenção Integral na Saúde da Família. Diário Oficial da União 2005; 5 jul.

9. Brasil. M inistério da Saúde. Portaria n 687 de 30 de março de 2006. Aprova a Política Nacional de Promoção da Saúde. Diário Oficial da U nião 2006; 31 mar.

10. Brasil. M inistério da Saúde. Portaria no 154 de 24 de janeiro de 2008. Cria os Núcleos de Apoio à Saúde da Família (NASF). Diário Oficial da União 2008; 4 mar.

11. Rebelatto JR. 0 objetivo de trabalho em fisioterapia e perspectivas de atuação e de ensino [dissertacão]. São Carlos (SP): Universidade Federal de São Carlos; 1986.

12. Pereira FWA, Mangueira JO, M onteiro MPA, Véras M MS, Lima VCS, Barrocas TCP, et al. A inserção da Fisioterapia na Estratégia Saúde da Família em Sobral/CE. SANARE [periódico na Internet] 2004 [acessado 2007 jul 23];V(1): [cerca de 8 p.]. Disponível em: http://www.sobral.ce.gov.br/ saudedafamilia/

13. Véras M M S, Pinto VPT, Oliveira EN, Quinderé PHD. O fisioterapeuta na Estratégia Saúde da Família: primeiros passos na construção de um novo modelo de atenção. SANARE [periódico na Internet] 2004 [acessado 2007 jul 23];V(1):[cerca de 5 p.] Disponível em: http://www.sobral.ce.gov.br/ saudedafamilia
14. Brasil ACO, Brandão JAM, Silva MON, GondinFilho VC. O papel do fisioterapeuta do programa saúde da família do município de Sobral-Ceará. RBPS 2005; 18(1):3-6.

15. Trelha CS, Silva DW, Lida LM, Fortes MH, Mendes TS. O fisioterapeuta no Programa de Saúde da Família de Londrina (PR). Revista Espaço para a Saúde 2007; 8(2):20-25.

16. Ramos LR. Fatores determinantes do envelhecimento saudável em idosos residentes em centro urbano: Projeto Epidoso, São Paulo. Cad Saude Publica 2003; 19(3):793-798.

17. Lima-Costa M F, Barreto SM, Giatti L. Condições de saúde, capacidade funcional, uso de serviços e gastos com medicamentos da população idosa brasileira: um estudo descritivo baseado na Pesquisa Nacional por Amostra de Domicílios. Cad Saude Publica 2003; 19(3):735-743.

18. Davim RM B, Torres GV, Dantas SM M, Lima VM . Estudo com idosos de instituições asilares no município de $\mathrm{Natal} / \mathrm{RN}$ : características socioeconômicas e de saúde. Rev. Latino-Am Enfermagem 2004; 12(3):18-24.

19. Rosa TEC, Benício MHA, Latorre MRDO, Ramos $L R$. Fatores determinantes da capacidade funcional entre idosos. Rev. Saude Publica 2003; 37(1):40-48.

20. Parahyba MI, Veras R, M elzer D. Incapacidade funcional entre as mulheres idosas no Brasil. Rev. Saude Publica 2005; 39(3):383-391.

21. Piccini RX, Facchini LA, Tomasi E, Thumé E, Silveira DS, Siqueira FV, Rodrigues MA. Necessidades de saúde comuns aos idosos: efetividade na oferta e utilização em atenção básica à saúde. Cien Saude Colet 2006; 11(3):657-667.

22. Costa N eto MM, Silvestre JA. A tenção à saúde do Idoso - Instabilidade Postural e Queda. 1a ed. Brasília: M inistério da Saúde; 2000.

23. Buss PM. Promoção da Saúde e Qualidade de Vida. Cien Saude Colet 2000; 5(1):163-177.

24. Gomes L. Fatores de risco e medidas profiláticas nas pneumonias adquiridas na comunidade. J Pneumol 2001; 27(2):97-114.

25. França LHG, Tavares V. Insuficiência Venosa Crônica. Uma atualização. J Vasc Br 2003; 2(4):318-328.

Artigo apresentado em 22/01/2008

Aprovado em 30/06/2008

Versão final apresentada em 29/07/2008 